\title{
Das Potenzial der Kinderliteratur im Hinblick auf den frühen Fremdspracherwerb \author{
in Early Language Learning
} \\ The Role of Children's Literature
}

\begin{abstract}
Children's literature is undoubtedly a very useful tool in teaching and learning foreign languages and its educational value is remarkable. Storybooks contain rich and authentic examples of the language being learned, they involve children personally and actively in the process of language learning and they address the issues relevant to children's interests and their perception of the world. The aim of the article is to show how children's literature can be used effectively in teaching a foreign language.
\end{abstract}

KEYWORDS: teaching and learning foreign languages, early language learning, children's literature, storybooks.

\section{LITERATUR UND FREMDSPRACHENUNTERRICHT}

Literatur hat im Leben eines jeden Menschen, egal ob Groß oder Klein, einen unbestrittenen Platz. Die Literatur aber schätzen zu lernen und genießen zu können bedarf seiner Zeit und einer gewissen Leseerfahrung. Erste Erfahrungen sammeln die Kinder in der Regel in ihren Familien, z.B. wenn ihnen Gute-Nacht-Geschichten erzählt oder vorgelesen werden. Aus der Unterrichtspraxis wird deutlich, dass solche Aktivitäten ein Meilenstein in der Förderung der späteren sprachlichen Kompetenz eines Kindes sind. Dank dem regelmäßigen Einsatz von Literatur lernen die Kinder nach und nach dem komplexen Sprachangebot einen Sinn entnehmen. Das betrifft 
sowohl den muttersprachlichen als auch den fremdsprachlichen Unterricht. Die schulische Praxis zeigt jedoch, dass der Literatur wieder einmal, wie es schon in den vergangenen Jahrzehnten oftmals der Fall war, der gebührende Platz im Fremdsprachenunterricht nicht gewährt wird und wenn man sich mit ihr im Unterricht beschäftigt, dann eher akzidentell.

Literatur ist ein umfangreicher Begriff, der das Schrifttum jeder Art impliziert, darunter also auch alle sprachlich fixierten nichtkünstlerischen Werke wie Kochrezepte oder Gesetze. Im engeren Sinne wird Literatur als „Ordnungsbegriff zur Klassifizierung einer Hauptkunstart benutzt, die neben anderen (bildende Kunst, Musik, Architektur etc.) existiert" (Biechele 2010a: 202). In der Geschichte des Fremdsprachenlehrens und -lernens wurde den literarischen Texten unterschiedliche Bedeutung beigemessen. Kast (1994: 5) konstatiert: „Literatur und Fremdsprachenunterricht, das ist die Geschichte der schüchternen Liebe und die Geschichte eines ständigen Mißbrauchs.". Bis heute bleiben viele Fragen rund um den Literatureinsatz im Fremdsprachenunterricht offen. Die bereits 1984 von Löschmann und Schröder formulierten Fragenstellungen sind auch in der modernen Fremdsprachendidaktik unbeantwortet:

Das betrifft die Einbindung des literarischen Textes in einen kommunikativ orientierten Fremdsprachenunterricht, die Aufdeckung der Möglichkeiten und Grenzen seines Einsatzes, die Bestimmung von Funktion und Stellenwert des Textes, seine Rolle sowohl bei der fremdsprachigen Könnens- und Persönlichkeitsentwicklung als auch bei der Verwirklichung der landeskundlichen Komponente im Unterricht und die Spezifika der Gestaltung von Aufgaben und Übungen zu literarischen Texten. (Löschmann, Schröder 1984: 7)

Dieser Beitrag will daran anknüpfen und einen theoretischen Rahmen für die gestellten Fragen bieten. Im Vordergrund der Überlegungen soll der junge Lerner stehen und die Möglichkeiten des Literatureinsatzes hinsichtlich des frühen Spracherwerbs. Kriterien der Textauswahl sowie didaktischmethodisches Vorgehen sollen nicht unberücksichtigt bleiben.

Eingangs soll unterstrichen werden, dass für das Fach Deutsch als Fremdsprache auf allen Bildungsstufen in Polen es weder einen Kanon an literarischen Texten gibt, noch sind die Lehrwerkautoren dazu verpflichtet, die Lerner mit den fremdsprachigen literarischen Texten vertraut zu machen. Im Referenzrahmen für Sprachen ist dagegen ein expliziter Umgang mit der Literatur erst ab der Niveaustufe B2 vorgesehen. Wo noch in den Lehrwerken der 80er und 90er Jahre etliche literarische Texte in die Lehrwerke integriert wurden (vgl. u.a. Sichtwechsel, Stufen international oder Themen neu), so ist gegenwärtig eine Tendenz zu verzeichnen, sich im Lehrund Lernprozess immer weniger anspruchsvoller Texte zu bedienen. Dieje- 
nigen, die bleiben, werden "kommunikativ ausgeschlachtet" (Kast 1994), sind der grammatischen, seltener der lexikalischen Progression untergeordnet. Das Bildungsziel des fremdsprachlichen Unterrichts besteht selbstverständlich primär in der Vermittlung sprachlicher Fähigkeiten und Fertigkeiten. Die moderne Schule hat aber darüber hinaus die Erziehungsziele zu realisieren, zu denen Fremdverstehen, Selbsterfahrung und Interkulturalität gehören (vgl. Biechele 2010b: 203). Diese Ziele können gerade dank dem Einsatz der Literatur im Deutschunterricht erfolgreich erreicht werden, denn literarische Texte wirken nach Löschmann und Schröder (1984: 18) bildend und erziehend zugleich. Sie erweitern den Erfahrungsbereich, bereichern die Gefühlswelt, regen die Phantasie an, steigern die Erlebnisfähigkeit (vgl. Löschmann, Schröder 1984: 18). Wenn die Alltagsdialoge im Anfängerunterricht nicht über ,Hallo-wie-heißt-du-Dialoge' hinausgehen, kann das Interesse an weiterem Fremdsprachenlernen in den weiterführenden Bildungsstufen erlahmen:

Wenn durchgehend Alltagsdialoge im Mittelpunkt des Lerngeschehens stehen, so wichtig sie auch immer für die Entwicklung einer Sprechhandlungskompetenz sein mögen, ergeben sich motivationale Probleme für den Lerner. Es ist nämlich frustrierend, wenn man sich mit der für einen Anfänger üblichen Langsamkeit und Anstrengung durch einen Text arbeitet und am Ende feststellen muss, dass dieser Text die Mühe nicht wert war, da er nur Banalitäten preisgibt. (Huneke, Steinig 2005: 84)

Literarische Texte können dabei erfolgreich auf allen Niveaustufen des Deutschunterrichts eingesetzt werden. Diejenigen Lehrer, die sich an die Kinderliteratur bereits im Frühbeginn wagen, werden bestimmt einen Mehrwert für den ganzen Lernprozess verzeichnen.

\section{KINDERLITERATUR UND DER KINDLICHE (FREMD-)SPRACHERWERB}

Der Begriff Kinder- und Jugendliteratur umfasst für O'Sullivan und Rösler (2002: 5) „eine Adressatengruppe vom Säugling bis hin zum jungen Erwachsenen und ein Korpus unterschiedlicher Texte wie Bilderbücher für Kleinstkinder, Erstlesebücher für Erstklässler, Lyriksammlungen für bereits literaturfähige Kinder und anspruchsvolle Adoleszenzromane". Kinderliteratur wird auch als Anfängerliteratur bzw. Literatur für literarische Anfänger bezeichnet, da wie O'Sullivan (2002: 11) bemerkt, „mit ihr die ersten Schritte im Prozess des Literaturerwerbs getätigt werden, der als Prozess der Aneignung von Regeln von Literatur und als Aufbau von literarischer Rezeptionskompetenz durch eine Progression von einfachen $\mathrm{zu}$ immer 
komplexeren Formen zu verstehen ist". O'Sullivan (2002: 11) betont dabei, dass das wesentliche Merkmal dieser Literatur - die Einfachheit - nicht mit Einfältigkeit oder Dürftigkeit zu verwechseln sei. Ausgehend von den Bedürfnissen der Kinder nach Emotionalität und Sicherheit auf der einen Seite, und dem Handlungsbedürfnis und der Experimentierfreude andererseits, sollen die eingesetzten Texte den Kindern die Möglichkeit bieten, sich kreativ, ohne Leistungsdruck auszuprobieren. Besonders die Bilderbücher können vielfältig im frühen Fremdsprachenunterricht eingesetzt werden. Sie bilden die optimale Grundlage für den Einstieg in ein literarisches Leben und „auch wenn Lesefähigkeiten und Fremdsprachenkenntnisse noch gering sind, können Leseinteressen und Lesegewohnheiten entwickelt und der eigenständige Umgang mit Literatur geschult werden" (Geyer 2011: 47).

Ein zielgruppenadäquat eingesetzter Text birgt viele didaktische und pädagogische Potentiale; er kann den Leser motivieren, für das Land, dessen Sprache man lernt, interessieren und vor allem anregen, mit dem Text kreativ umzugehen. Die größte Bedeutung hat er aber wohl für den Spracherwerb.

Wenn wir nach der anthropozentrischen Theorie von Sprache und Spracherwerb annehmen, dass sprachliche Fähigkeiten weder ,erworben' noch ,übertragen' werden, sondern von konkreten Menschen entwickelt generiert werden müssen (vgl. Grucza F. 2010: 258), dann ist Bleyhl (2000: 112) nur zuzustimmen, wenn er meint, der Unterricht habe von Anfang an dem Kriterium der 'kritischen Masse' an Spracherfahrung zu entsprechen. Es ist aber nicht die Quantität der Reize, die den Erfolg beim Spracherwerb ausmacht, nicht die unentwegte Sprachberieselung (Butzkamm 1993: 11) ist entscheidend, sondern das Aufnehmen voll verstandener Sprache in sinnvollen Lebenskontexten:

Being exposed to language is not like being exposed to a virus. One doesn't catch it automatically... The learner must do something active that involves him cognitively in the process. (Seliger 1977: 275, in: Butzkamm 1993: 11)

Um eine Fremdsprache erfolgreich zu lehren und zu lernen genügt der Einsatz des Lehrwerkes nicht. Bleyhl (2000: 112) macht darauf aufmerksam, das Lehrbuch biete im Allgemeinen zu wenig Sprache, die oftmals dazu in reduzierter Form präsentiert wird. Der traditionellste Typ der Stoffverteilung im Lehrbuch ist die grammatische/strukturelle Progression, in der sich der Lernstoff an sprachsystematischen Gesichtspunkten orientiert, wodurch die Sprachkompetenz zwar flexibel und ausbaufähig entwickelt wird, die Sprache in den Lehrbuchtexten sich jedoch dann als unnatürlich erweist. „Nur dank der Teilnahme an Kommunikationsprozessen, also der Auseinandersetzung mit sprachlichen Äußerungen (Texten) können die Lernen- 
den, sowohl das sprachliche (und nichtsprachliche) Wissen rekonstruieren, als auch die Fähigkeiten entwickeln, sprachliche Äußerungen zu Kommunikationszwecken anzuwenden." (Olpińska-Szkiełko 2012: 451) Auch Skowronek (2011: 133) betont die besondere Rolle, die beim Spracherwerb den Texten zukommt: „Die einzige Möglichkeit, die Sprachkompetenz zu entwickeln, ist der Umgang mit Texten einer Sprache, also das Initiieren der Prozesse des Verstehens und der Produktion von Diskurs.". Wir dürfen nicht vergessen, dass das Ziel jedes Fremdsprachenunterrichts die schrittweise Entwicklung der sprachlichen sowie der Kommunikationsfähigkeiten (vgl. Grucza S. 2004: 75) jedes einzelnen Lernenden ist. Wie Skowronek (2011: 129) also zu Recht betont, Sprachen sollen für kommunikative Zwekke unterrichtet und gelernt werden. Und hier öffnet sich das Potenzial der Kinderbücher, die, so Geyer (2011: 47), sowohl thematisch als auch sprachlich auf die Lebenswelt von Kindern zugeschnitten sind und eignen sich deshalb auch besonders gut zur Erweiterung des Wortschatzes. Von Vorteil erweisen sich wiederkehrende Sprachmuster, die einerseits Verständnisschwierigkeiten überwinden lassen, andererseits sich leicht einprägen und nachspielen lassen. Das Bilderbuch ist so konzipiert, dass es die Kinder weder unter- noch überfordert. Und gerade die einfachen, sich wiederholenden sprachlichen Strukturen laden zum spielerischen Umgang mit dem Text ein.

Was noch im Zusammenhang mit den Bilderbüchern zu betonen gilt, effektive Lernhilfe leisten meistens hochqualitative, liebevoll aufbereitete Bilder oder Zeichnungen. Diese können dann als Handlungsanlass (nonverbaler oder verbaler Art - wie die Kommunikation eben ist) dienen. Denn die Wissenspsychologie macht darauf aufmerksam, das Wissen entsteht aus der physischen oder mentalen Aktivität eines Individuums, wobei es in die Handlungsschemata des Einzelnen eingegliedert wird (vgl. Bleyhl 2000: 110). Spätestens seit Piaget ist es ersichtlich, dass das Verstehen eine nicht zu unterschätzende Rolle für den Spracherwerbsprozess hat: Eine Sprache lernt man am besten, wenn man sie auch gebraucht und man auf diesem Wege Rückmeldungen über Sinnkonstruktionen bekommt. Kast (1994: 8) spricht im Zusammenhang mit dem handlungsorientierten Lernen von der Einlösung der Forderung learning by doing, von einem schüler- und handlungsorientierten Literaturunterricht mit der prominenten Rolle der Verfahren und Methoden, „in denen Schülerinnen und Schüler, ausgehend von ihren Erfahrungen, ihren Vorstellungen, ihrem Wissen mit dem und am Text Handlungen durchführen, d.h. mit dem Text "etwas machen“ - und in denen nicht (nur) über den Text geredet wird“. Einfache Geschichten, wie sie meistens in den Bilderbüchern dargestellt werden, bieten zahlreiche Möglichkeiten, die Kinder aktiv in die Erzählung einzubinden und tragen somit 
dem kindlichen Bedürfnis sich mitzuteilen Rechnung: von non-verbalen Aktivitäten für die noch nicht sprechreifen Kinder wie Bebilderung (einer Szene aus) der Geschichte, pantomimisches Nachspielen, über Wortschatzspiele bis hin zum Nacherzählen. Damit sich die Kinder als Sprachhandelnde erleben können, müssen die Inhalte für die Kinder relevant sein und dicht an ihre individuellen Erfahrungswelten anknüpfen. Von großem Belang sind auch die Interaktions- oder Rollenspiele, die den Kindern die Möglichkeit bieten, das vorhin Gelernte zu internalisieren und kommunikativ anzuwenden (vgl. Lewicka, Lewicki 2004: 169). Denn das, was man als Handelnder erlebt, wird auch nachhaltiger behalten.

Literatureinsatz kann auch Interesse für die Kultur des Zielsprachenlandes wecken. Eine Sprache ist ja unauflöslich mit der Kultur der Zielsprachengemeinschaft verbunden, fast jedes Wort einer Sprache ist "kulturell konnotiert, manche stärker, andere schwächer" (Huneke, Steinig 2005: 68). Wenn man eine Sprache lernt, möchte man auch etwas über die Menschen, die diese Sprache sprechen, ihre Kulturen, ihre Länder erfahren. Wenn wir die Neugier der kleinen Lerner ernst nehmen, schafft das gemeinsame Lesen vielfältige Entfaltungsmöglichkeiten für die Kinder:

Im Gespräch über Bilder und Gehörtes wird das Kind je nach Entwicklungsstufe aktiv: Es kann benennen und nachfragen, gemeinsam wird in der Kleingruppe ein Begriff umschrieben, neu gedeutet, Assoziationen aus dem individuellen Leben der Kinder werden angeregt und geäußert, es wird vermutet, spekuliert, fortgeführt, zurückgeblättert und phantasiert. (Vogl 2006: 19)

Im Elementarbereich lautet die Devise nicht „Übung macht den Meister", somit erübrigen sich und entfallen routinierte Nachsprechübungen, die weder den Kindern Spaß machen noch sie geistig engagieren. Das Spielerische, das gemeinsam Erlebte ebnet den Weg der erfolgreichen Sprachreise. Nicht ohne Bedeutung für die Motivation zum weiteren Fremdsprachenlernen bleibt das Erfolgserlebnis: Die Kinder müssen nicht jedes einzelne Wort verstehen - das Sprachverstehen erfolgt aus dem Kontext, unterstützt durch Tonfall, Gestik und Mimik (vgl. Bonacchi 2011, Vogl 2006: 19) oder viele Bilder.

Auch wenn uns scheinbar nicht viel Zeit im Unterricht zur Verfügung steht, anspruchsvollere Aufgaben zu realisieren, so sollten wir als Lehrer davor jedoch nicht scheuen, dieses auszuprobieren. Die herausfordernden Aufgaben sind es diejenigen, die den Kindern Freude bereiten und letztendlich auch die Kinder motivieren. Hoppenstedt (2011: 9) betont, „Vor allem in einem nur mit einem spärlichen Zeitfenster ausgestatteten frühen Fremdsprachenunterricht sollten die motivierenden und sensibilisierenden Gesichtspunkte Vorrang haben, die für eine positive Einstellung zur Fremd- 
sprache und Selbstvertrauen im Umgang mit ihr führen.". Durch den Einsatz geeigneter literarischer Texte kann dieser Forderung Rechnung getragen werden.

\section{KRITERIEN DER TEXTAUSWAHL}

Für die Auswahl von Texten im Frühen Fremdsprachenunterricht empfiehlt Beirat Frühes Deutsch (2011: 4) u.a. folgende Kriterien in Erwägung zu ziehen:

1. Texte sollten multimodal sein, d.h. unterschiedliche Sinnesorgane ansprechen.

2. Texte sollten einen visuellen Anteil haben, d.h. statische oder bewegte Bilder enthalten.

3. Texte sollten einen kollaborativen Ansatz haben, d.h. sie sollten dem Leser Möglichkeiten zur Zusammenarbeit bieten.

Im Zusammenhang mit dem Postulat nach Authentizität soll auf die Neudefinierung hingewiesen werden. Das Prinzip der Authentizität im Sinne von Sambor Grucza kommt in Texten zum Tragen, die in dem Fremdsprachenerwerbsprozess zur Entwicklung fremdsprachlicher Kompetenz führen, es umfasst also sowohl primär für den Fremdsprachenunterricht produzierte Texte, als auch solche, die nicht primär für den Fremdsprachenunterricht produziert, sondern die für den Bedarf fremdsprachlichen Unterrichts adaptiert wurden (vgl. Grucza S. 2000: 79, ausführlich zum Authentizitätspostulat in der Glottodidaktik s. u.a. Grucza S. 2004). Heute wird das Prinzip der Authentizität auf die gesamte Unterrichtssituation erweitert. Nach Edelhoff (1985) spricht man von der „pädagogischsituativen Authentizität", einer Lernerorientierung also, in der die Handlungen an die Bedürfnisse sowie kognitive Leistungsmöglichkeiten der Kinder angepasst werden. „Ein authentischer früher Fremdsprachenunterricht in diesem Sinn bewirkt eine Vielzahl von Kommunikationssituationen, die für das Kind bedeutsam sind, weil es sich ganz persönlich angenommen und angesprochen fühlt." (Hoppenstedt 2011: 9) Für den glottodidaktischen Prozess bedeutet das folglich, "dass die Lernenden von ihrem Gesichtspunkt aus relevante und authentische Aufgaben und Übungen erfüllen sollten, dank derer sie ihre Kommunikationserfahrung gewinnen, sammeln und bereichern können und die ihnen bei der Wahrnehmung realer Kommunikationsaufgaben in realen Kommunikationssituationen nützlich und behilflich sein können" (Olpińska-Szkiełko 2012: 451).

Eine detaillierte Kriterienliste für die Textauswahl im Fremdsprachenunterricht lieferte bereits 1990 Gerhard Neuner. Für die Beurteilung der 
Eignung eines Textes zieht der Autor vier Aspekte hervor: den Aspekt der Lern- und Leseziele, der Lernvoraussetzungen, des Lerngegenstandes sowie den Aspekt der Lernsituation. Gembus (2011: 157) dagegen stellt einen Fragenkatalog zur Geschichtenauswahl für den Primarbereich zusammen. Folgende Fragen sollen bei der Wahl eines Adressaten geeigneten Textes hilfreich sein:

Bietet die Geschichte...

- Möglichkeiten zur emotionalen Auseinandersetzung?

- eine abwechslungsreiche Handlung?

- inhaltliche und/oder sprachliche Wiederholungen?

- Typen statt individueller Charaktere?

- einen Bezug zum Alltag der Kinder in Verbindung mit Außergewöhnlichem/ Geheimnisvollen?

Entscheidendes Kriterium ist, das Kind soll sich von der Geschichte angesprochen fühlen. Um das zu erreichen, sollen Beziehungen zwischen den Erfahrungen und Erlebnissen der Kinder und den Geschichten hergestellt werden (vgl. Apeltauer 2004: 154). Außer dem Bezug zur Kinderwelt empfiehlt Geyer (2011: 50) aber auch besonders auf Sprache und Text zu achten: "Neben einem begrenzten Textumfang pro Seite sollte das Buch in gut lesbarer Druckschrift geschrieben sein und kurze, einfache Sätze enthalten." Auch die Bilder gilt es zu bewerten. Geyer (2011: 50) bemerkt, diese sollten „nicht nur dekorativ, sondern auch aussagekräftig sein und das Verständnis der Geschichte wesentlich unterstützen.".

\section{METHODISCH-DIDAKTISCHES VORGEHEN}

Vom Einsatz literarischer Texte kann auch der frühe DaF-Unterricht profitieren. Ein derartiger Unterricht muss allerdings den besonderen Lernvoraussetzungen dieser Altersstufe Rechnung tragen. Bei den jungen Lernern muss mit einer geringen Konzentrationsspanne gerechnet werden. Der frühe Fremdsprachenunterricht muss daher besonders viel Abwechslung und Bewegungsraum schaffen, um dem Bewegungsdrang der Kinder Rechnung zu tragen. Die Arbeitsmethoden im Elementarbereich sollen bedeutend von denen mit den Jugendlichen und Erwachsenen divergieren. Lernprozesse sollen so geplant und realisiert werden, dass die ganze Person mit all ihren kognitiven, emotionalen, sozialen und expressiven Möglichkeiten angesprochen und gefördert wird (vgl. Legutke 2010: 117). Ähnlich wie im muttersprachlichen Unterricht, in dem die Kinder motiviert werden ihre Ausdrucksfähigkeiten und insbesondere ihr sprachliches Repertoire zu entwickeln und zu erweitern (vgl. Apeltauer 2004: 155), sollten im frühen 
Fremdsprachenunterricht genügend Anlässe geboten werden, eigene Ideen und Vorstellungen einzubringen. Solche Aufgaben wie z.B. ein eigenes Bilderbuch erstellen, die vorgelesene Geschichte pantomimisch nachspielen, ein Theaterstück vorführen oder den Text verändern animieren einerseits die Kinder zum handelnden Lernen, andererseits lockern das Unterrichtsgeschehen auf. Auch das interaktive Vorlesen oder Erzählen einer Geschichte und die gemeinsame Ausführung der anschließenden Aktivitäten beugen der Routine vor und befreien vom jeglichen Leistungsdruck.

Damit die Kinder von der Arbeit mit literarischen Texten profitieren, müssen die Lehrkräfte bestimmte Prinzipien befolgen:

- Kurze Sätze - langsames Vorlesen - damit die Kinder genügend Zeit haben, sich die Geschichte vorzustellen, aufgrund bestimmter Merkmale sie zu ordnen.

- Soweit es möglich ist, die Geschichte mit realen Gegenständen untermauern, z.B. die Spieltiere, (Hand-)Puppen als Helden spielen lassen, ein Tuch für die Landschaft verwenden, etc.

- Schon beim Vorlesen die Kinder in den Erzählprozess einbeziehen und sie mitwirken lassen - Kinder können bestimmte Geräusche, Bewegungen erfolgreich inszenieren.

- Bilder helfen - unter der Voraussetzung sie sind anschaulich, eindeutig und verwirren nicht.

- Wenn die Geschichte länger ist, können auch Pausen eingelegt werden, in denen auf den Inhalt eingegangen wird und evtl. auch in Beziehung mit der Erfahrung der Kinder zusammengebracht wird, z.B. Wer von euch war schon... / hat schon... (zur sprachlichen Entlastung kann das auch ab und zu in der Muttersprache erfolgen).

- Es muss nicht immer eine neue Geschichte vorgelesen werden, die Kinder können immer wieder dieselbe Geschichte hören, wenn sie ihnen gefällt. Von Vorteil erweist sich dann in dieser Situation, dass sie nach und nach immer öfter in den Text sprachlich eingreifen können.

- Auch auf einem niedrigen sprachlichen Niveau sollten wir den Kindern Anlässe schaffen, sprachlich zu handeln. Einzelne Sätze aus der Geschichte können als Modell für die eigene Sprachproduktion dienen.

Zahlreiche Ideen, wie Schüler im Anfangsunterricht an Texte ,Hand anlegen' können, werden u.a. von Kast (1994: 10) und Hölscher (2007: 157) zusammengestellt. Die didaktische Aufbereitung und methodische Umsetzung eines Texteinsatzes im Unterricht werden von Klasse zu Klasse stark variieren, zumal die Aufgaben sich nach den Lernern und ihren emotionalen, kognitiven, kreativen und sozialen Fähigkeiten richten sollten. Eines ist 
jedoch gemeinsam: eine interessante Lektüre kann den Brückenschlag zwischen Spaß und Lernen schaffen und was Iluk (2011: 224) zusätzlich betont,

durch den inhaltlich bedeutungsvollen Lernstoff [...] bekommen die Schüler die reale Möglichkeit, ihre Aufmerksamkeit auf den Inhalt zu richten, Hypothesen zu bilden und sie zu verifizieren. Dadurch können sie zunächst eine kritische Masse an lexikalischen Einheiten bzw. an Spracherfahrungen erreichen, die notwendig für einen weiteren, beschleunigteren und folglich effektiveren Spracherwerb ist und wodurch die Kinder folglich auch mehr von dem Sprachangebot dauerhaft behalten.

Wenn sprachliche Übungen vorgeschlagen werden, so gilt es grundsätzlich $\mathrm{zu}$ berücksichtigen, dass die sprachlichen Formen von Anfang an sprachlichen Interaktionen dienen und sie bereichern (vgl. Olpińska-Szkiełko 2012: 453).

\section{ABSCHLIESSENDE BEMERKUNGEN}

Ein kreativer Umgang impliziert oftmals zugleich einen Eingriff in einen literarischen Text, was wiederum viele Kontroversen hervorrufen kann. Kast (1994: 8) lässt die Frage danach, ob das Eingreifen in den literarischen Text „kein Sakrileg an jenem sprachlichen Kunstwerk, dem man sich mit Respekt, Ehrfurcht und Bescheidenheit nähern sollte" mit den Worten des Schriftstellers Hans Magnus Enzensberger beantworten:

Der Leser hat ... immer recht, und es kann ihm niemand die Freiheit nehmen, von einem Text den Gebrauch zu machen, der ihm paßt. Zu dieser Freiheit gehört es, hin- und herzublättern, ganze Passagen zu überspringen, Sätze gegen den Strich zu lesen, sie mißzuverstehen, sie umzumodeln, sie fortzuspinnen und auszuschmücken mit allen möglichen Assoziationen, Schlüsse aus dem Text zu ziehen, von denen der Text nichts weiß, sich über ihn zu ärgern, sich über ihn zu freuen, ihn zu vergessen, ihn zu plagiieren und das Buch, worin er steht, zu einem beliebigen Zeitpunkt zu verwerfen. Die Lektüre ist ein anarchischer Akt. Die Interpretation, besonders die einzig richtige, ist dazu da, diesen Akt zu vereiteln. (Enzensberger 1988)

Wenn nach Gembus (2011: 161f.) die im Unterricht präsentierten Geschichten auch kritisch hinterfragt werden und die Möglichkeit bieten, bei den jungen Lernern Denkprozesse anzustoßen, „die über reine Erfassung des Inhalts hinausgehen“, dann bietet so ein Unterricht „weit mehr als eine Möglichkeit, die Fremdsprache im Kontext zu präsentieren“. Wir führen die Kinder an eine Leserwelt heran, die sich ihnen in Zukunft in all ihrer Breite bietet und mit jeder neugelesenen Lektüre neue (sprachliche) Erfahrungen sammeln lässt. 


\section{LITERATURVERZEICHNIS}

Apeltauer, E., 2004. Oralität, Literalität und Medienkompetenz. In: Badstübner-Kizik, C., Rozalowska-Żądło, R., Kniszewska, A. (Hrsg.). Sprache lehren, Sprache lernen. Festschrift für Prof. Halina Stasiak. Gdańsk: 147-167.

Beirat Frühes Deutsch, 2011. Arbeit mit authentischen Texten. Möglichkeiten und Grenzen für den frühen Fremdsprachenunterricht. In: Frühes Deutsch. Heft 22, 4-5.

Biechele, W., 2010a. Literatur. In: Barkowski, H., Krumm, H.-J. (Hrsg.). Fachlexikon Deutsch als Fremd- und Zweitsprache. Tübingen-Basel: Francke, 202.

Biechele, W., 2010b. Literaturdidaktik. In: Barkowski, H., Krumm, H.-J. (Hrsg.). Fachlexikon Deutsch als Fremd- und Zweitsprache. Tübingen-Basel: Francke, 203.

Bleyhl, W., 2000. Fremdsprachenlernen konstruktiv. In: Wendt, M. (Hrsg.). Konstruktion statt Instruktion: neue Zugänge zu Sprache und Kultur im Fremdsprachenunterricht. Frankfurt am Main: Peter Lang, 103-117.

Bonacchi, S., 2011. Höflichkeitsausdrücke und anthropozentrische Linguistik. Warszawa: EuroEdukacja.

Butzkamm, W., 1993. Psycholinguistik des Fremdsprachenunterrichts. Tübingen-Basel: Francke.

Edelhoff, Ch., 1985. Authentizität im Fremdsprachenunterricht. In: Edelhoff, Ch. (Hrsg.) Authentische Texte im Fremdsprachenunterricht. Einführung und Unterrichtsmodelle. München: Hueber Verlag, 7-30.

Gembus, A., 2011. Unterrichten mit Geschichten: Ein Modell zur Unterrichtsplanung mit narrativen Formen und dessen Anwendung. In: Kötter, M., Rymarczyk, J. (Hrsg.). Fremdsprachenunterricht in der Grundschule. Forschungsergebnisse und Vorschläge zu seiner weiteren Entwicklung. Frankfurt am Main: Peter Lang, 153-162.

Geyer, S., 2011. Bilderbücher im DaF-Unterricht der Primarstufe. In: Frühes Deutsch. Heft 22, 47-50.

Grucza, F., 2010. Zum ontologischen Status menschlicher Sprachen, zu ihren Funktionen, den Aufgaben der Sprachwissenschaft und des Sprachunterrichts. In: Kwartalnik Neofilologiczny, LVII, 257-274.

Grucza, S., 2000. Kommunikative Adäquatheit glottodidaktischer Texte - zur Kritik des sog. Authentizitätspostulats. Adekwatność komunikacyjna tekstów glottodydaktycznych krytyka tzw. postulatu autentyczności. In: Niemiecki w Dialogu/ Deutsch im Dialog, 2/1, 73-103.

Grucza, S., 2004. Autentyczność i oryginalność tekstów a glottodydaktyczne nieporozumienia w tej sprawie. In: Przeglad Glottodydaktyczny, 19, 75-83.

Hölscher, P., 2007. Lernszenarien. Sprache kann nicht gelehrt, sondern nur gelernt werden. In: Ahrenholz, B. (Hrsg.). Deutsch als Zweitsprache. Voraussetzungen und Konzepte für die Förderung von Kindern und Jugendlichen mit Migrationshintergrund. Breisgau: Welke Fillibach Verlag, 151-167.

Hoppenstedt, G., 2011. Authentizität und der Einsatz authentischer Materialien und Medien im frühen Fremdsprachenunterricht. In: Frühes Deutsch, Heft 22, 6-9.

Huneke, H.-W., Steinig, W., 2005. Deutsch als Fremdsprache. Eine Einführung. Berlin: Erich Schmidt Verlag.

Iluk, J., 2011. Der Narrative Ansatz im frühen Fremdsprachenunterricht und seine Effizienz. In: Adamczak-Krysztofowicz, S., Kowalonek-Janczarek, M., Maciejewski, M., Sopata, A. Aktuelle Probleme der Angewandten Linguistik. Interkulturalität als Schlüsselkompetenz für Fremdsprachenlehrer, Übersetzer und Mediatoren. Poznań: Wydawnictwo Naukowe UAM, 217-225.

Kast, B., 1994. Literatur im Anfängerunterricht. In: Fremdsprache Deutsch, Heft 11, 4-13. 
Legutke, M., 2010. Holismus. In: Barkowski, H., Krumm, H.-J. (Hrsg.). Fachlexikon Deutsch als Fremd- und Zweitsprache. Tübingen-Basel: Francke, 117.

Lewicka, G., Lewicki, R., 2004. Zur Rolle des Spiels in der Fremdsprachenfrüherziehung. In: Badstübner-Kizik, C., Rozalowska-Żądło, R., Uniszewska, A. (Hrsg.). Sprachen lehren Sprachen lernen. Festschrift für Halina Stasiak zum 70. Geburtstag. Gdańsk, 167-175.

Löschmann, M., Schröder, G., 1984. Literarische Texte im Fremdsprachenunterricht. Leipzig: VEB Verlag.

Olpińska-Szkiełko, M., 2012. Glottodidaktische Implikationen der anthropozentrischen Sprachentheorie. In: Olpińska-Szkiełko, M., Grucza, S., Berdychowska, Z., Żmudzki, J. (Hrsg.). Der Mensch und seine Sprachen. Festschrift für Professor Franciszek Grucza. Frankfurt am Main: Peter Lang, 445-454.

O'Sullivan, E., 2002. Aktuelles Fachlexikon. In: Fremdsprache Deutsch, Heft 27, 10-11.

O'Sullivan, E., Rösler, D., 2002. Kinder- und Jugendliteratur im Deutschunterricht. Fremdsprache Deutsch, Heft 27, 5-8.

Skowronek, B., 2011. Deutsch als Fremdsprache: ökokratisches Konzept. In: Glottodidactica, XXXVII, 129-140.

Vogl, I., 2006. Lese-Paten. In: Frühes Deutsch, Heft 6, 19-21. 